\title{
Atividade antimicrobiana dos extratos metanólicos de diferentes espécies do gênero
}

\section{Lippia}

\author{
Antimicrobial activity of methanol extracts from different species of the genus Lippia \\ Actividad antimicrobiana de extractos metanólicos de diferentes especies del género Lippia
}

Recebido: 09/07/2021 | Revisado: 14/07/2021 | Aceito: 15/07/2021 | Publicado: 24/07/2021

Soraya Castro Trindade

ORCID: https://orcid.org/0000-0001-7125-9114

Universidade Estadual de Feira de Santana, Brasil

E-mail: soraya@uefs.br

Jurandi Nery Pereira-Filho

ORCID: https://orcid.org/0000-0001-8577-5734

Universidade Estadual de Feira de Santana, Brasil

E-mail: jurandinery@gmail.com

Laerte Oliveira Barreto Neto

ORCID: https://orcid.org/0000-0001-9090-9063

Universidade Estadual de Feira de Santana, Brasil

E-mail: laertebarreto9@gmail.com

Thais Brito de Oliveira Moura

ORCID: https://orcid.org/0000-0002-3084-9422

Universidade Federal da Bahia, Brasil

E-mail: thais.britoliveira@gmail.com

Antonio Pedro Fróes de Farias

ORCID: https://orcid.org/0000-0002-2625-9470

Universidade Federal da Bahia, Brasil

E-mail: froes_pedro@hotmail.com

José Tadeu Raynal Rocha Filho

ORCID: https://orcid.org/0000-0002-2771-0235

Universidade Federal da Bahia, Brasil

E-mail: jtraynal@hotmail.com

Rebeca Pereira Bulhosa Santos

ORCID: https://orcid.org/0000-0002-9625-5117

Universidade Federal da Bahia, Brasil

E-mail: biorebecabulhosa@gmail.com

Acsa Oliveira Magalhães

ORCID: https://orcid.org/0000-0003-4141-2637

Universidade Estadual de Feira de Santana, Brasil E-mail: acsaomagalhaes@hotmail.com

Ellen Karla Nobre dos Santos-Lima

ORCID: https://orcid.org/0000-0001-5633-8260

Centro Universitário Mario Ponte Jucá, Brasil E-mail: ellenobre@hotmail.com

Paulo Cirino Carvalho-Filho

ORCID: https://orcid.org/0000-0002-1006-6471

Escola Bahiana de Medicina e Saúde Pública, Brasil

E-mail: pauloccfilho@gmail.com

Vera Lúcia Costa Vale

ORCID: https://orcid.org/0000-0002-4055-5049

Universidade do Estado da Bahia, Brasil

E-mail: vvale@uneb.br

Angélica Maria Lucchese

ORCID: https://orcid.org/0000-0003-0751-762X

Universidade Estadual de Feira de Santana, Brasil

E-mail: angelica.lucchese@gmail.com

\section{Resumo}

As espécies do gênero Lippia (Verbenaceae) possuem diversas moléculas bioativas, oriundas do seu metabolismo secundário, que apresentam atividade antimicrobiana, analgésica, antifúngica e imunomoduladora. Este estudo teve como objetivo avaliar a atividade antimicrobiana in vitro do extrato metanólico das folhas de L. alnifolia, L. origanoides, L. insignis e L. thymoides frente a microrganismos de importância clínica. Foi realizada uma triagem fitoquímica dos extratos das plantas e a avaliação da atividade antimicrobiana pelo método de difusão em disco, além da determinação da concentração inibitória mínima (MIC) frente às bactérias Bacillus subtilis, Escherichia coli, Micrococcus luteus, 
Pseudomonas aeruginosa, Staphylococcus aureus e Salmonella choleraesuis, e os fungos Candida albicans e Candida glabrata. A presença de saponinas, terpenos, esteroides, cumarinas, ácidos fenólicos e flavonoides foi observada na análise fitoquímica. Os extratos metanólicos das folhas de L. alnifolia, L. origanoides e L. insignis apresentaram atividade antimicrobiana contra todas os microrganismos testados. O extrato metanólico da L. thymoides apresentou atividade antimicrobiana frente aos microrganismos $M$. luteus, $P$. aeruginosa, $S$. aureus, C. albicans e $C$. glabrata. A maioria dos extratos apresentou atividade bacteriostática e todos os extratos apresentaram atividade fungistática para $C$. albicans e fungicida para C. glabrata. A utilização dos extratos de Lippia pode ser uma alternativa viável no tratamento de doenças infecciosas de origem bacteriana e fúngica.

Palavras-chave: Lippia; Antimicrobianos; Extratos.

\begin{abstract}
The species of the genus Lippia (Verbenaceae) have several bioactive molecules from their secondary metabolism, which have antimicrobial, analgesic, antifungal and immunomodulatory activities. This study aimed to evaluate the in vitro antimicrobial activity of methanol extract of $L$. alnifolia, L. origanoides, L. insignis and L. thymoides leaves, against clinically important microorganisms. A phytochemical screening of plant extracts was carried out and the antimicrobial activity was evaluated by the disk diffusion method, in addition to the determination of the minimum inhibitory concentration (MIC) against the bacteria Bacillus subtilis, Escherichia coli, Micrococcus luteus, Pseudomonas aeruginosa, Staphylococcus aureus Salmonella choleraesuis, and the fungi Candida albicans and Candida glabrata. The presence of saponins, terpenes, steroids, coumarins, phenolic acids and flavonoids was observed in the phytochemical evaluation. Methanolic extracts from the leaves of L. alnifolia, L. origanoides and L. insignis showed antimicrobial activity against all tested microorganisms. The methanol extract of L. thymoides showed antimicrobial activity against the microorganisms M. luteus, P. aeruginosa, S. aureus, C. albicans and C. glabrata. Most extracts showed bacteriostatic activity and all extracts showed fungistatic activity for $C$. albicans and fungicide for C. glabrata. The use of Lippia extracts can be a viable alternative in the treatment of bacterial and fungal infectious diseases.
\end{abstract}

Keywords: Lippia; Antimicrobials; Extracts.

\title{
Resumen
}

Las especies del género Lippia (Verbenaceae) tienen varias moléculas bioactivas, derivadas de su metabolismo secundario, que tienen actividad antimicrobiana, analgésica, antifúngica e inmunomoduladora. Este estudio tuvo como objetivo evaluar la actividad antimicrobiana in vitro del extracto metanólico de hojas de L. alnifolia, L. origanoides, $L$. insignis y L. thymoides contra microorganismos clínicamente importantes. Se realizó un cribado fitoquímico de extractos vegetales y se evaluó la actividad antimicrobiana por el método de difusión en disco, además de la determinación de la concentración mínima inhibitoria (CIM) contra las bacterias Bacillus subtilis, Escherichia coli, Micrococcus luteus, Pseudomonas aeruginosa, Staphylococcus aureus y Salmonella choleraesuis y los hongos Candida albicans y Candida glabrata. Se observó la presencia de saponinas, terpenos, esteroides, cumarinas, ácidos fenólicos y flavonoides en el análisis fitoquímico. Los extractos metanólicos de las hojas de L. alnifolia, L. origanoides y L. insignis teníam actividad antimicrobiana contra todos los microorganismos analizados. El extracto metanólico de $L$. thymoides mostró actividad antimicrobiana contra los microorganismos M. luteus, P. aeruginosa, S. aureus, C. albicans y C. glabrata. La mayoría de los extractos tenían actividad bacteriostática y todos los extractos teníam actividad fungistática para $C$. albicans y fungicida para $C$. glabrata. El uso de extractos de Lippia puede ser una alternativa viable en el tratamiento de enfermedades infecciosas bacterianas y fúngicas.

Palabras clave: Lippia; Antimicrobianos; Extractos.

\section{Introdução}

O uso de produtos naturais tem se tornado uma alternativa importante para o tratamento de diversas doenças, por apresentarem compostos e moléculas bioativas, provenientes principalmente do seu metabolismo secundário, que possuem atividade antimicrobiana, analgésica, antifúngica e imunomoduladora (Juiz, Alves \& Barros, 2010; Palaska, Papathanasiou \& Theoharides, 2013). Neste cenário, espécies do gênero Lippia (Verbenaceae), de ocorrência do Brasil, particularmente do Nordeste, se constituem em uma importante fonte de classes e compostos químicos que apresentam propriedades medicinais, como terpenos, timol, carvacrol, linalol, limoneno e óxido de piperitenona, podendo ter efeitos positivos em diversas doenças (Hirota et al., 2010; Juiz et al., 2015; Oliveira et al., 2018; Rozza et al., 2011).

Dentre as espécies do gênero Lippia, L. origanoides Kunth, conhecida popularmente como "alecrim-pimenta” ou "salvade-marajó", é utilizada popularmente para o tratamento de diversas afecções (Costa, Souza, Brito \& Fontenelle, 2017; LeyvaJiménez, Lozano-Sánchez, Cádiz-Gurrea, Arráez-Román \& Segura-Carretero, 2019). Possui ampla distribuição no Brasil e faz 
parte da lista das plantas medicinais de uso do Sistema Único de Saúde (SUS) (Brasil, 2009). Esta variedade possui ação antibacteriana, antiespasmódica, anti-inflamatória e antioxidante, decorrente dos seus constituintes fenólicos e não-fenólicos (Botelho et al., 2007; Okhale, Nwanosike, Fatokun \& Kunle, 2016; Oliveira, Leitão, Fernandes \& Leitão, 2014; Sarrazin et al., 2015). Embora seja uma das espécies de Lippia mais estudadas (Botelho et al., 2008, 2016; Oliveira, Oliveira, Carneiro, Silva \& Costa, 2018), o conhecimento sobre as propriedades biológicas dos seus compostos ainda não está sedimentado e, portanto, ainda possui um grande potencial biotecnológico a ser explorado.

Lippia insignis Moldenke está listada no Livro Vermelho da Flora do Brasil como uma espécie sujeita a extinção, por ser vulnerável à antropização da região, que afetou a qualidade do seu habitat (Martinelli \& Moraes, 2013). A literatura sobre as propriedades químicas, farmacológicas e biológicas dessa espécie de Lippia ainda é escassa e necessita de melhores estudos para avaliar o seu potencial biotecnológico. Ela é caracterizada por ser endêmica do Brasil, encontrada principalmente no estado da Bahia, no bioma caatinga. Sendo assim, em consonância com a Estratégia Nacional para Conservação Ex Situ de Espécies Ameaçadas da Flora Brasileira, torna-se necessário a realização de estudos que demonstrem o potencial para o uso medicinal de Lippia insignis, ajudando, inclusive, na sua preservação.

A espécie L. thymoides Mart. \& Schauer, conhecida popularmente como "alecrim-do-mato" ou "alecrim-do-campo", tem despertado o interesse de pesquisadores (Oliveira, Leitão, Fernandes \& Leitão, 2014; Silva et al., 2015), por ser promissora para a indústria farmacêutica e de defensivos. Além disso, a atividade antimicrobiana de seus óleos essenciais e extratos tem sido demonstrada por meio de testes qualitativos e quantitativos (Pinto et al., 2013; Rodrigues, Tavares, Pereira \& Costa, 2009; Silva et al., 2015). Já Lippia alnifolia Mart. \& Schauer conhecido como "alecrim-do-mato", "alecrim-de-vaqueiro" e "pedrécio", é utilizado na medicina popular como antisséptico e para tratar doenças que afetam o aparelho respiratório, como bronquite e asma (Vilela et al., 2020). A ação antimicrobiana de extratos de folhas, caules e inflorescências foi relatada frente a Staphylococcus aureus e Candida parapsilosis (Pinto et al, 2013), entretanto foi uma avaliação restrita ao teste de difusão em disco, justificando assim uma ampliação do estudo.

Por outro lado, a utilização de agentes antimicrobianos convencionais no combate a doenças infecciosas é uma fonte crescente de preocupação, uma vez que a resistência microbiana se tornou um problema de saúde pública no mundo (Meira, Todescan, Azoubel, Bittencourt \& Azoubel, 2007; Juiz, Alves \& Barros, 2010; Tavares et al., 2020). O emprego indiscriminado de antimicrobianos favoreceu o aparecimento de linhagens multirresistentes, tornando necessário a adoção de medidas de controle. Sendo assim, o objetivo deste estudo foi avaliar a atividade antimicrobiana dos extratos metanólicos de diferentes espécies do gênero Lippia contra microrganismos de importância clínica.

\section{Material e métodos}

\subsection{Coleta e obtenção dos extratos}

A coleta das folhas de Lippia insignis Moldenke e Lippia origanoides Kunth foi realizada em 15 de maio e 28 de agosto de 2013, respectivamente, no município de Feira de Santana, Bahia, em banco de germoplasma do Horto Florestal da Universidade Estadual de Feira de Santana (UEFS). As exsicatas das espécies foram depositadas no Herbário da UEFS sob os códigos HUEFS83373 (L. origanoides) e HUEFS197674 (L. insignis). As folhas de Lippia alnifolia foram coletadas no Pico das Almas, em Rio de Contas (Bahia), em 13 de setembro de 2013. Uma exsicata foi depositada no herbário da UEFS (HUEFS 213618). Já a coleta do material vegetal de Lippia thymoides Mart \& Schauer, foi realizada em novembro de 2015, na Coleção de Plantas Medicinais e Aromáticas da Unidade Experimental do Horto Florestal. Uma exsicata da espécie oriunda desta coleção está depositada no Herbário da UEFS, sob número de registro 241404. 
Após separação das inflorescências e caules, as folhas foram secas em temperatura ambiente e ao abrigo da luz e em seguida foram pulverizadas em moinho de facas. Procedeu-se, então, a extração consecutiva por maceração com metanol (relação 1:3, material vegetal: metanol), por três vezes, obedecendo o intervalo de cinco dias entre cada extração. Por fim, o ex trato bruto das folhas de cada planta foi reunido e concentrado em evaporadores rotatórios, originando o extrato metanólico, que foi armazenado em geladeira para posteriores análises.

\subsection{Triagem Fitoquímica}

Para a avaliação dos constituintes ativos presentes nos extratos, foram avaliados os perfis cromatográficos em camada delgada (CCD). Utilizando-se capilares de vidro, as amostras foram aplicadas em placas (5 X $20 \mathrm{~cm}$ ) com base de alumínio revestidas com sílica gel 60 com indicador de fluorescência (Alugram Sil G/UV ${ }_{254}$ - Macherey-Nagel, Alemanha). As cromatoplacas foram ativadas em estufa a $120^{\circ} \mathrm{C}$ por 30 minutos antes de adicionar a amostra. Em seguida, o material foi eluido em cubas cromatográficas, empregando-se o sistema de solventes diclorometano-acetona-metanol (80:18:2) como eluente.

Após eluição, as placas foram secas, visualizadas em luz UV-254 nm e $365 \mathrm{~nm}$ e reveladas com os reagentes anisaldeído-ácido sulfúrico (AS), dragendorff com ácido clorídrico (DRG), Liebermann-Burchard (LB), hidróxido de potássio (KOH) e Produtos Naturais-Polietilenoglicol (NP/PEG) (Wagner \& Bladt, 1995). A coloração dos cromatogramas revelados foi comparada com as figuras presentes no Atlas de Wagner e Bladt (1995).

A presença de saponinas foi observada por meio do ensaio qualitativo de formação de espuma (Matos, 1997). Uma amostra do extrato foi dissolvida em ácido clorídrico $(\mathrm{HCl})$ concentrado e em seguida $10 \mathrm{~mL}$ de água destilada foram adicionados. A solução foi filtrada em um tubo de ensaio e agitada vigorosamente por dois a três minutos, observando-se, então a formação e a duração da espuma.

\subsection{Avaliação do potencial antimicrobiano}

\subsubsection{Preparação do extrato para a realização dos testes}

O extrato das folhas das espécies do gênero Lippia foram diluídas em metanol (Synth, Diadema, SP, Brasil) / DMSO (Dimetilsulfóxido) (Synth) (1:3) a 3\% e filtradas com membrana estéril de 0,22 $\mu$ m para obtenção das alíquotas para os testes.

Alíquotas dos extratos preparadas das folhas foram submetidas a dosagem de proteínas com a técnica de Lowry modificada, de acordo com as instruções do fabricante (DC ${ }^{\text {TM }}$ Protein Assay da Biorad, CA, USA) e os resultados foram obtidos pela leitura em espectrofotômetro.

\subsubsection{Difusão em Disco}

A avaliação do potencial antimicrobiano foi efetuada de acordo com a norma M2-A8 do National Committee for Clinical Laboratory Standards / Agência Nacional de Vigilância Sanitária (NCCLS, 2003). As linhagens microbianas utilizadas no presente trabalho são pertencentes a American Type Culture Collection (ATCC). Foram utilizadas as bactérias Bacillus subtilis (ATCC 6633), Escherichia coli (ATCC94863), Micrococcus luteus (ATCC10240), Pseudomonas aeruginosa (ATCC 15442), Staphylococcus aureus (ATCC 6538), Salmonella choleraesuis (ATCC14028) e os fungos, Candida albicans (ATCC18804) e Candida glabrata (CCT0728).

As cepas bacterianas foram cultivadas por 24 horas em meio Ágar Mueller Hilton (Himedia, Índia) e os fungos por 48 horas em Ágar Sabouraud Dextrose (Kasvi, Itália). Os microrganismos foram incubados em estufa bacteriológica a $37^{\circ} \mathrm{C}$. Em seguida, as colônias dos microrganismos foram transferidas para tubos contendo $9 \mathrm{~mL}$ de solução salina (0,9\%), que, após homogeneizado, foi comparado ao tubo padrão 0,5 da escala McFarland ( $\left.1,5 \times 10^{8} \mathrm{UFC} / \mathrm{mL}\right)$. Após a padronização, as culturas foram espalhadas uniformemente na superfície das placas de meio de cultura com auxílio de um swab estéril. Discos de $6 \mathrm{~mm}$ 
contendo $10 \mu \mathrm{L}$ do extrato das diferentes espécies do gênero Lippia $(0,1 \mathrm{mg} / \mathrm{mL})$ foram colocados em triplicata. Como controle, foram utilizados discos impregnados com DMSO, Cloranfenicol (Laborclin, SP, Brasil) (30 $\mu$ g) e/ou Ciclopiroxolaminam (Mupirox) (EMS, SP, Brasil). As placas contendo os discos foram incubadas em estufa bacteriológica a $37^{\circ} \mathrm{C}$ por $24-48 \mathrm{~h}$. A leitura foi realizada mensurando o diâmetro os halos inibitórios formados ao redor das bactérias e fungos, com o uso de paquímetro.

\subsubsection{Concentração inibitória mínima (MIC)}

A determinação da concentração inibitória mínima (MIC) foi baseada no método de microdiluição em caldo proposta pelo Clinical and Laboratory Standards Institute, Norma M100-S15 (CLSI, 2005). As linhagens bacterianas utilizadas foram Bacillus subtilis (ATCC 6633), Escherichia coli (ATCC 94863), Micrococcus luteus (ATCC 10240), Pseudomonas aeruginosa (ATCC 15442), Staphylococcus aureus (ATCC 6538), Salmonella choleraesuis (ATCC14028) e os fungos, Candida albicans (ATCC18804) e Candida glabrata (CCT0728).

Para a realização do ensaio, foram distribuídos $100 \mu \mathrm{L}$ / poço do meio de cultura Broth Nutriente (Himedia, Índia) em placa de 96 poços estéril e o mesmo volume do extrato metanólico das diferentes espécies do gênero Lippia, em diferentes concentrações $(1,0-0,01 \mathrm{mg} / \mathrm{mL})$, obtido através de sucessivas diluições seriadas (1:1), em triplicata. Como controles, foram utilizados DMSO a 1\%, Cloranfenicol para bactérias e Ciclopiroxolaminam para os fungos. Os microrganismos foram previamente cultivados em meio de cultura, como descrito no item anterior. Foram distribuídos $100 \mu \mathrm{L}$ de suspensão dos diferentes microrganismos em cada poço, exceto na última fileira (continha apenas o meios de cultura). Após as inoculações, a placa foi incubada em estufa bacteriológica a $37^{\circ} \mathrm{C}$, por 24 (para as bactérias), ou 48 horas (para os fungos). Após esse período, uma alíquota do poço com a menor concentração inibitória foi plaqueada em meio de cultura para avaliar se os extratos apresentavam efeito bacteriostático/bactericida ou fungistático/fungicida.

\section{Resultados}

\subsection{Triagem fitoquímica}

Nos extratos obtidos das folhas de foi observada uma quantidade de espuma persistente e abundante, indicando a ocorrência de saponinas. A triagem fitoquímica por cromatografia em camada delgada de todos extratos indicou a presença de alguns compostos das classes de terpenos, esteroides, cumarinas, ácidos fenólicos e flavonoides. A presença de alcaloides não foi detectada.

\subsection{Avaliação do potencial antimicrobiano}

\subsubsection{Difusão em disco}

Os resultados do teste de difusão em disco, usado para avaliar a atividade antimicrobiana dos extratos estão demonstrados na Tabela 1. 
Tabela 1. Halos de inibição (em milímetros) dos microrganismos pelo extrato metanólico das folhas de diferentes espécies do gênero Lippia obtidos com o teste de difusão em disco.

\begin{tabular}{lcccccc}
\hline \multirow{2}{*}{ Microrganismo } & \multicolumn{5}{c}{ Halos de inibição (mm) } \\
\cline { 2 - 6 } & L. alnifolia & L. origanoides & L. insignis & L. thymoides & CN & CP \\
\hline B. subtilis (ATCC 6633) & - & - & - & - & - & 30 \\
E. coli (ATCC 94863) & - & - & - & - & - & 24 \\
M. luteus (ATCC 10240) & $(11,67 \pm 1,25)$ & $(9,67 \pm 0.47)$ & $(14,03 \pm 0,05)$ & - & - & 35 \\
& & & & & & \\
P. aeruginosa (ATCC 15442) & $(7,67 \pm 0,94)$ & $(9 \pm 0,058)$ & $(8 \pm 0,82)$ & $(8,33 \pm 0,58)$ & - & 25 \\
S. aureus (ATCC 6538) & - & - & - & $(9,66 \pm 0,58)$ & - & 25 \\
S. choleraesuis (ATCC14028) & $(8 \pm 0,82)$ & $(10,67 \pm 1,25)$ & $(10,67 \pm 0,94)$ & $(7,33 \pm 1,53)$ & - & 23 \\
Candida albicans (ATCC18804) & - & $(22 \pm 1,63)$ & $(11,67 \pm 1,70)$ & $(11,33 \pm 2,31)$ & - & 22 \\
Candida glabrata (CCT0728) & - & - & - & - & - & 30 \\
\hline
\end{tabular}

CN - Controle Negativo, CP - Controle Positivo, - Sem atividade. Fonte: Autores.

Os extratos metanólicos das folhas de Lippia alnifolia, Lippia origanoides, Lippia insignis e L. thymoides induziram a formação de halos de inibição frente às bactérias Pseudomonas aeruginosa e Salmonella choleraesuis. Destes, três extratos apresentaram atividade antimicrobiana contra a bactéria Micrococcus luteus (L. alnifolia, L. origanoides e L. insignis). Apenas o extrato da planta L. thymoides foi capaz de formar halo de inibição contra a bactéria Staphylococcus aureus. Nenhum dos extratos testados foi capaz de apresentar atividade antimicrobiana no teste de difusão em disco frente aos microrganismos Bacillus subtilis e Escherichia coli na concentração de $0,1 \mathrm{mg} / \mathrm{mL}$.

Com relação à atividade fungicida, os extratos das plantas Lippia origanoides, Lippia insignis e L. thymoides apresentaram atividade antimicrobiana frente a levedura $C$. albicans. Nenhum dos extratos testados foi capaz de formar halos de inibição frente a levedura C. glabrata $(0,1 \mathrm{mg} / \mathrm{mL})$.

\subsubsection{Concentração inibitória mínima (MIC)}

Os dados dos ensaios de determinação da concentração inibitória mínima estão apresentados na Tabela 2.

Os extratos metanólicos das folhas de L. alnifolia, L. origanoides e L. insignis apresentaram atividade antimicrobiana contra todas as bactérias de importância clínica testadas, Gram-positivas e Gram-negativas, além dos fungos $C$. albicans $e$ C. glabrata. O extrato metanólico da L. thymoides apresentou atividade antimicrobiana frente aos microrganismos $M$. luteus, $P$. aeruginosa, S. aureus, C. albicans e C. glabrata. Apesar do extrato de L. thymoides ter induzido a formação de um pequeno halo de inibição no teste de difusão em disco contra a bactéria $S$. choleraesuis $(7,33 \pm 1,53 \mathrm{~mm})$, o MIC revelou que essa bactéria foi capaz crescer mesmo na concentração $1,0 \mathrm{mg} / \mathrm{mL}$ do extrato. 
Tabela 2. Concentração inibitória mínima $(\mathrm{mg} / \mathrm{mL})$ dos extratos metanólicos das folhas de diferentes espécies do gênero Lippia contra microrganismos de importância clínica.

\begin{tabular}{lcccccccc}
\hline \multirow{2}{*}{ Microrganismo } & \multicolumn{2}{c}{ L. alnifolia } & \multicolumn{2}{c}{ L. origanoides } & \multicolumn{2}{c}{ L. insignis } & \multicolumn{2}{c}{ L. thymoides } \\
\cline { 2 - 8 } & MIC & Efeito & MIC & Efeito & MIC & Efeito & MIC & Efeito \\
\hline B. subtilis (ATCC 6633) & 1,0 & $*$ & 1,0 & $*$ & 1,0 & $*$ & - & - \\
E. coli (ATCC 94863) & 1,0 & $*$ & 1,0 & $*$ & 1,0 & $*$ & - & - \\
M. luteus (ATCC 10240) & 0,5 & $*$ & 0,5 & $*$ & 0,5 & $*$ & 1,0 & $\#$ \\
P. aeruginosa (ATCC 15442) & 0,25 & $*$ & 0,125 & $*$ & 0,25 & $*$ & 0,031 & $*$ \\
S. aureus (ATCC 6538) & 1,0 & $*$ & 0,25 & $*$ & 0,25 & $*$ & 1,0 & $\#$ \\
S. choleraesuis (ATCC14028) & 0,25 & $*$ & 0,5 & $*$ & 0,25 & $*$ & - & - \\
Candida albicans (ATCC18804) & 0,063 & $\circ$ & 0,125 & $\circ$ & 0,5 & $\circ$ & 0,031 & $\circ$ \\
Candida glabrata (CCT0728) & 0,25 & + & 0,25 & + & 0,5 & + & 0,25 & + \\
\hline
\end{tabular}

\# bactericida; * bacteriostático; + fungicida; ${ }^{\circ}$ fungistático. - Sem atividade. Fonte: Autores.

Com relação ao tipo de atividade, se bactericida ou bacteriostática, a maioria dos extratos metanólicos apresentaram atividade bacteriostática frente as linhagens bacterianas testadas. O extrato metanólico da L. thymoides apresentou atividade bactericida contra Micrococcus luteus e Staphylococcus aureus. E todos os extratos apresentaram atividade fungistática para Candida albicans e fungicida para Candida glabrata.

\section{Discussão}

A quantidade de espuma persistente e abundante, indicou a presença de saponinas, constituintes encontrados no gênero Lippia (Costa, Souza, Brito \& Fontenelle, 2017; Palaska, Papathanasiou \& Theoharides, 2013). As saponinas são consideradas adjuvantes na atividade moduladora da resposta imune, principalmente na indução da polarização das células $T$ e apresentação de antígenos (Costa et al., 2005; Marques et al., 2015; Nunes-Pinheiro, Leite, Farias, Braga \& Lopes, 2003), o que pode indicar o potencial uso dos extratos avaliados em estratégias preventivas e terapêuticas relacionadas a imunomodulação.

A triagem fitoquímica dos extratos detectou a presença de compostos das classes de terpenos, esteroides, cumarinas, ácidos fenólicos e flavonoides. Este resultado já era esperado, uma vez que essas classes de compostos são pertencentes ao gênero Lippia, como já identificados ou isolados em estudos anteriores (Botelho et al., 2016; Okhale, Nwanosike, Fatokun \& Kunle, 2016; Palaska, Papathanasiou \& Theoharides, 2013; Pascual, Slowing, Carretero, Sánchez Mata \& Villar, 2001; Pinto et al., 2013; Soares \& Tavares-Dias, 2013; Vilela et al., 2020). Entretanto, não foi observada a presença de alcaloides, o que pode estar relacionado ao modo e ao local da coleta das folhas, já que estes estão entre os fatores que podem influenciar na presença de determinados metabólitos secundários (Gobbo-Neto \& Lopes, 2007; Hirota et al., 2010; Santos et al., 2016).

Os extratos metanólicos das diferentes espécies de Lippia apresentaram atividade antibacteriana e antifúngica contra os microrganismos de importância clínica testados. L. origanoides é utilizada na medicina popular para o tratamento de diversas infecções (Leyva-Jiménez, Lozano-Sánchez, Cádiz-Gurrea, Arráez-Román \& Segura-Carretero, 2019) e sua ação antimicrobiana contra as linhagens testadas está de acordo ao relatado em trabalhos anteriores (Leal et al., 2019; Perera et al., 2017; Queiroz et al., 2014; Sarrazin et al., 2015). Para L. insignis este é o primeiro relato da atividade antimicrobiana frente a microrganismos de importância clínica, demonstrando o seu potencial uso biotecnológico. A espécie L. thymoides tem despertado o interesse de pesquisadores (Oliveira, Leitão, Fernandes \& Leitão, 2014; Silva et al., 2015) por ser uma fonte de produtos naturais de interesse 
farmacêutico (Silva et al., 2016) e por apresentar atividade antimicrobiana contra diversos microrganismos (Pinto et al., 2013; Rodrigues, Tavares, Pereira \& Costa, 2009; Silva et al., 2015), o que foi confirmado neste trabalho. Já para L. alnifolia, utilizada na medicina popular como antisséptico e com relatos anteriores de ação de extratos de folhas apenas frente a S. aureus (Pinto et al, 2013), observou-se, neste trabalho, ação antibacteriana e antifúngica. Cabe destacar que os extratos de $L$ alnifolia e $L$ thymoides apresentaram os melhores resultados de atividade inibitória frente a Candida albicans e Pseudomonas aeruginosa, demonstrando seu uso potencial na prática clínica.

No teste do antibiograma pelo método de difusão em disco, o extrato metanólico de Lippia thymoides formou halos de inibição contra a bactéria Salmonella choleraesuis, no entanto, o resultado do MIC demonstrou que o extrato não foi efetivo na inibição da Salmonella choleraesuis, mesmo em concentrações de $1,0 \mathrm{mg} / \mathrm{mL}$. Vale ressaltar que os testes de difusão em disco só podem obter resultados confiáveis no antibiograma quando as medidas do diâmetro do halo de inibição estão correlacionados às concentrações inibitórias mínimas (NCCLS, 2003), como realizado no presente estudo.

As plantas do gênero Lippia apresentam propriedades biológicas que fazem dela uma fonte natural para a produção de fitoterápicos (Costa, Souza, Brito \& Fontenelle, 2017). Os dados encontrados no presente trabalho, trazem potenciais alternativas para o tratamento de infecção bacterianas de importância clínica a fim de superar o surgimento de micro-organismos multirresistentes. Além disso, esses extratos podem servir de opções no tratamento de infecções oportunistas, como as causadas por fungos do gênero Candida. Existem, inclusive, testes e pesquisas intercalando seu potencial antimicrobiano e o desenvolvimento de fitoterápicos capazes de reduzir ou até mesmo transpor as resistências fúngicas e bacterianas através do uso combinado de produtos naturais com sintéticos já comercializados (Barreto et al., 2014; Costa, Souza, Brito \& Fontenelle, 2017).

\section{Considerações Finais}

A utilização dos extratos de Lippia, bem como dos seus compostos, pode ser uma alternativa viável no tratamento de doenças infecciosas de origem bacteriana e fúngica. Estudos devem ser desenvolvidos a fim de avaliar a ação consorciada dos diferentes extratos, isolar os compostos com atividade antimicrobiana e determinar sua eventual citotoxicidade em células humanas.

\section{Referências}

Barreto, H. M., Lima, I. S., Coelho, K. M. R. N., Osório, L. Â. R., Mourão, R. A., Santos, B. H. C., Coutinho, H. D. M., Abreu, A. P. L., Medeiros, M. G. F., Citó, A. M. G. L., \& Lopes, J. A. D. (2014). Effect of Lippia origanoides H.B.K. essential oil in the resistance to aminoglycosides in methicillin resistant Staphylococcus aureus. Eur J Integr Med, 6, 560-564.

Botelho, M. A., Barros, G., Queiroz, D. B., Carvalho, C. F., Gouvea, J., Patrus, L., Bannet, M., Patrus, D., Rego, A., Silva, I., Campus, G., \& Araújo-Filho, I. (2016). Nanotechnology in phytotherapy: antiinflammatory effect of a nanostructured thymol gel from Lippia sidoides in acute periodontitis in rats. Phytother Res, 30, 152-159.

Botelho, M. A., Nogueira, N. A. P., Bastos, G. M., Fonseca, S. G. C., Lemos, T. L.G., Matos, F. J. A., Montenegro, D., Heukelbach, J., Rao, V. S., \& Brito, G. A. C. (2007). Antimicrobial activity of the essential oil from Lippia sidoides, carvacrol and thymol against oral pathogens. Braz J Med Biol Res, $40,349-356$.

Botelho, M. A., Rao, V. S., Montenegro, D., Bandeira, M. A. M., Fonseca, S. G. C., Nogueira, N. A. P., Ribeiro, R. A., \& Brito, G. A. C. (2008). Effects of a herbal gel containing carvacrol and chalcones on alveolar bone resorption in rats on experimental periodontitis. Phytother Res, $22,442-449$.

Brasil. (2009). Relação nacional de plantas medicinais de interesse ao SUS. Ministério da Saúde.

Clinical and Laboratory Standards Institute. (2005). Performance standards for antimicrobial susceptibility testing, fifteenth informational supplement. CLSI document M100-S15. Wayne, Pensylvania: CLSI.

Costa, J. G. M., Rodrigues, F. F. G., Angélico, E. C., Silva, M. R., Mota, M. L., Santos, N. K. A., \& Lemos, T. L. G. (2005). Estudo químico-biológico dos óleos essenciais de Hyptis martiusii, Lippia sidoides e Syzigium aromaticum frente às larvas do Aedes aegypti. Rev Bras Farmacogn, 15(4), 304-309.

Costa, P. S., Souza, E. B., Brito, E. H. S., \& Fontenelle, R. O. S. (2017). Atividade antimicrobiana e potencial terapêutico do gênero Lippia sensu lato (Verbenaceae). Hoehnea, 44(2), 158-171.

Gobbo-Neto, L., \& Lopes, N. P. (2007). Plantas medicinais: fatores de influência no conteúdo de metabólitos secundários. Quim Nova, 30(2), $374-381$. 
Hirota, T., Lee, J. W., Lewis, W. G., Zhang, E. E., Breton, G., Liu, X., Garcia, M., Peters, E. C., Etchegaray, J. P., Traver, D., Schultz, P. G., \& Kay, S. A. (2010). High-throughput chemical screen identifies a novel potent modulator of cellular circadian rhythms and reveals CKI $\alpha$ as a clock regulatory kinase. PLoS Biol, 8(12), 1-14.

Juiz, P. J. L., Alves, R. J. C., \& Barros, T. F. (2010). Uso de produtos naturais como coadjuvante no tratamento da doença periodontal. Rev Bras Farmacogn, 20(1), 134-139.

Juiz, P. J. L., Lucchese, A. M., Gambari, R., Piva, R., Penolazzi, L., Di Ciano, M., Uetanabaro, A. P. T., Silva, F., \& Avila-Campos, M. J. (2015). Essential oils and isolated compounds from Lippia alba leaves and flowers: antimicrobial activity and osteoclast apoptosis. Int J Mol Med, 35(1), 211-217.

Leal, A. L. A. B., Braga, A. L., Barbosa, B. A., Rodrigues, A. M. X., Teixeira, F. A. F. C., Silva, K. M. R., Júnio, E. C., Leite, T. R. S., \& Amorim, M. S. (2019). Antimicrobial action of essential oil of Lippia origanoides H.B.K. J Clin Microbiol Biochem Technol, 5(1), 07-12.

Leyva-Jiménez, F. J., Lozano-Sánchez, J., Cádiz-Gurrea, M. L., Arráez-Román, D., \& Segura-Carretero, A. (2019). Functional ingredients based on nutritional phenolics. A case study against inflammation: Lippia genus. Nutrients, 11(1646), 1-24.

Marques, G. S., Silva, C. C. A. R., Vilela, W. T., Figueirêdo, C. B. M., Silva, A. C. A. F., Silva, R. M. F., \& Neto, P. J. R. (2015). Plantas medicinais como alternativa terapêutica para aumento da resistência imunológica. Rev Ciênc Farm Básica Apl, 36(1), 27-33.

Martinelli, G., \& Moraes, M. A. (2013). Livro vermelho da flora do Brasil.: Andrea Jakobsson: Instituto de Pesquisas Jardim Botânico do Rio de Janeiro.

Matos, F. J. A. (1997). Introdução à Fitoquímica Experimental. Edições UFC.

Meira, A. L. T., Todescan, S. M. C., Azoubel, E., Bittencourt, S., \& Azoubel, M. C. F. (2007). Uso de antimicrobianos locais em periodontia: uma abordagem crítica. Periodontia, 17(1), 92-98.

National Committee For Clinical Laboratory Standards. (2003). Performance standards for antimicrobial disk susceptibility tests, approved standard - eighth edition. NCCLS document M2-NCCLS. Wayne, Pennsylvania: NCCLS.

Nunes-Pinheiro, D. C. S., Leite, A. K. R. M., Farias, V. M., Braga, L. T., \& Lopes, C. A. P. (2003). Atividade imunomoduladora das plantas medicinais: perspectivas em medicina veterinária. Ciência Anim, 13(1), 23-32.

Okhale, S. E., Nwanosike, E. M., Fatokun, O. T., \& Kunle, O. F. (2016). Phytochemistry and ethnopharmacology of Lippia genus with a statement on chemotaxonomy and essential oil chemotypes. Int J Pharmacogn, 3(5), 201-211.

Oliveira, A. R. M. F., Oliveira, L. M., Carneiro, J. S., Silva, T. R. S., \& Costa, L. C. B. (2018). Leaf anatomy and essential oil production of Lippia native species. Brazilian J Agric, 93(3), 324-335.

Oliveira, D. R., Leitão, G. G., Fernandes, P. D., \& Leitão, S. G. (2014). Ethnopharmacological studies of Lippia origanoides. Rev Bras Farmacogn, 24, 206214.

Palaska, I., Papathanasiou, E., \& Theoharides, T. C. (2013). Use of polyphenols in periodontal inflammation. Eur J Pharmacol, 720, 77-83.

Pascual, M. E., Slowing, K., Carretero, E., Sánchez Mata, D., \& Villar, A. (2001). Lippia: traditional uses, chemistry and pharmacology: a review. J Ethnopharmacol, 76(3), 201-214.

Perera, W. H., Bizzo, H. R., Gama, P. E., Alviano, C. S., Salimena, F. R. G., Alviano, D. S., \& Leitão, S. G. (2017). Essential oil constituents from high altitude Brazilian species with antimicrobial activity: Baccharis parvidentata Malag., Hyptis monticola Mart. ex Benth. and Lippia origanoides Kunth. JEOR, 29(2), 109-116.

Pinto, C. P., Rodrigues, V. D., Pinto, F. P., Pinto, R. P., Uetanabaro, A. P. T., Pinheiro, C. S. R., Gadea, S. F. M., Silva, T. R. S., \& Lucchese, A. M. (2013). Antimicrobial activity of Lippia species from the Brazilian semiarid region traditionally used as antiseptic and anti-infective agents. Evid Based Complement Alternat Med, 2013, 1-5.

Queiroz, M. R. A., Almeida, A. C., Andrade, V. A., Lima, T. S., Martins, E. R., Figueiredo, L. S., \& Careli, R. T. (2014). Avaliação da atividade antibacteriana do óleo essencial de Lippia origanoides frente à Staphylococcus sp. isolados de alimentos de origem animal. Rev Bras Pl Med, 16(3), 737-743.

Rodrigues, Í. S. C., Tavares, V. N., Pereira, S. L. S., \& Costa, F. N. (2009). Antiplaque and antigingivitis effect of Lippia sidoides. A double-blind clinical study in humans. J Appl Oral Sci, 17(5), 404-407.

Rozza, A. L., Moraes, T. M., Kushima, H., Tanimoto, A., Marques, M. O. M., Bauab, T. M., Hiruma-Lima, C. A., \& Pellizzon, C. H. (2011). Gastroprotective mechanisms of Citrus lemon (Rutaceae) essential oil and its majority compounds limonene and $\beta$-pinene: involvement of heat-shock protein-70, vasoactive intestinal peptide, glutathione, sulfhydryl compounds, nitric oxide and prostaglandin $\mathrm{E}_{2}$. Chemico-Biological Interactions, 189, 82-89.

Santos, N. O., Pascon, R. C., Vallim, M. A., Figueiredo, C. R., Soares, M. G., Lago, J. H. G., \& Sartorelli, P. (2016). Cytotoxic and antimicrobial constituents from the essential oil of Lippia alba (Verbenaceae). Medicines, 3(22), 1-9.

Sarrazin, S. L. F., Silva, L. A., Oliveira, R. B., Raposo, J. D. A., Silva, J. K. R., Salimena, F. R. G., Maia, J. G. S., \& Mourão, R. H. V. (2015). Antibacterial action against food-borne microorganisms and antioxidant activity of carvacrol-rich oil from Lippia origanoides Kunth. Lipids Health Dis, 14(145), 1-8.

Silva, F. S., Menezes, P. M. N., Sá, P. G. S., Oliveira, A. L. S., Souza, E. A. A., Bamberg, V. M., Oliveira, H. R., Oliveira, S. A., \& Araújo, R. E., Uetanabaro, A. P. T., Silva, T. R. S., Almeida, J. R. G. S. \& Lucchese, A. M. (2015). Pharmacological basis for traditional use of the Lippia thymoides. Evid Based Complement Alternat Med, 2015, 1-10 
Research, Society and Development, v. 10, n. 9, e22610918051, 2021

(CC BY 4.0) | ISSN 2525-3409 | DOI: http://dx.doi.org/10.33448/rsd-v10i9.18051

Silva, F. S., Menezes, P. M. N., Sá, P. G. S., Oliveira, A. L. S., Souza, E. A. A., Almeida, J. R. G. S., Lima, J. T., Uetanabaro, A. P. T., Silva, T. R. S., Peralta, E. D., \& Lucchese, A. M. (2016). Chemical composition and pharmacological properties of the essential oils obtained seasonally from Lippia thymoides. Pharm Biol, 54(1), 25-34.

Soares, B. V., \& Tavares-Dias, M. (2013). Espécies de Lippia (Verbenaceae), seu potencial bioativo e importância na Medicina Veterinária e Aquicultura. Biota Amaz, 3(1), 109-123.

Tavares, L. S., Souza, V. C., Nunes, V. S., Silva, O. N., Souza, G. T., Marques, L. F., Goliatt, P. V. Z. C., Viccini, L. F., Franco, O. L., \& Santos, M. O. (2020). Antimicrobial peptide selection from Lippia spp leaf transcriptomes. Peptides, 129, 1-8.

Vilela, D. A. D., Silva, B. A. O., Brito, M. C., Menezes, P. M. N., Bomfim, H. F., Duarte-Filho, L. A. M. S., Silva, T. R. S., Ribeiro, L. A. A., Lucchese, A. M., \& Silva, F. S. (2020). Lippia alnifolia essential oil induces relaxation on Guinea-pig trachea by multiple pathways. J Ethnopharmacol, 246, 1-10.

Wagner, H., \& Bladt, S. (1995). Plant Drug Analysis: a thin layer chromatography atlas. Springer Verlag. 